\title{
COVID-19 is currently taking a toll on women's health
}

COVID-19 (SARS-cov-2), which was first reported in Wuhan, China on December 31, 2019, ${ }^{1}$ is currently taking a toll on women's health. While the global response has rightly focused on containing the virus and treating the infected, it also has gaps in our approach to women's health.

The causative agent of the outbreak, a new type of virus in the Coronaviridae family, spread rapidly resulting in an epidemic across China and the rest of the world. According to WHO, there are cases on all continents, except Antarctica; 202 affected countries are currently reported. ${ }^{2}$

The high ability to spread the infection of this new coronavirus has been shown to be through the secretions of the human body, mainly from the respiratory tract. To date, the time interval in which an individual infected with COVID-19 is infectious is unknown, ${ }^{3}$ due to the evidence that some studies have shown in asymptomatic individuals, they can continue to transmit the infection. ${ }^{4}$

Many countries continue with the recommendations established by the corresponding national and international organizations in health matters, which with a political support many demonstrate greater economic interest than in health care; parcels that initially denote the lack of sanitary surveillance protocols in biological agents, the same recommendations that due to the virulence of the pathogen, have not allowed an understanding of the pathophysiology and much less of the prevention of the disease, affecting the entire population's economy, mainly to the most vulnerable people.

The preventive measures of isolation, which continue to date, have led people to a consumption of economic resources that consequently affect the mental and physical health of our patients.

ACOG has issued practical advisory with information on how to optimize obstetric care in the midst of COVID- $19,{ }^{5}$ but... does the medical professional in public and private health have the resources to carry out these recommendations?

How does it affect female health?

Almost half a year after the COVID-19 pandemic, patients who, with fear of becoming infected, have postponed their health care, not only in gynecological, but in obstetric issues.

The doctor-patient relationship has been limited to informal communication, which with the use of different means of communication (text messages, calls or video calls), culminates through the interpretation of an apparent "accurate" diagnosis by the physician. Basically the number of consultations involving preventive medicine in the health area such as family planning methods, the timely detection of cervical-uterine and breast cancer, have been left aside.

\author{
Volume 9 Issue 3 - 2020 \\ Mario Alberto Garza-Garza \\ Fertility Center - IECH, Mexico
}

Correspondence: Mario Alberto Garza-Garza, Fertility Center - IECH, Monterrey, Av. Hidalgo 1842 Pte., 3er. Piso Colonia Obispado, C.P. 64060 Monterrey, N.L. Mexico, Tel 8I I5558I95, Emailmario.gza@gmail.com

Received: July 25, 2020 | Published: July 31, 2020

On the obstetric side, prenatal care has reduced its vigilance, valuing only those issues that, on the part of the patient, seem necessary or urgent; omitting or not detecting high-risk pregnancies that require closer monitoring by the ObGyn.

How much longer will preventive women's health continue to be postponed in this pandemic?

To date, there seems to be no treatment or vaccine already approved for the infection of COVID-19, for now we only have to carry on with the health measures, awaiting research to improve our outlook, in addition to generating new strategies towards recovering feminine preventive health.

\section{References}

1. Li Q, Guan X, Wu P, et al. Early transmission dynamics in Wuhan, China, of novel coronavirus-infected pneumonia. N Engl J Med. 2020.

2. Organization WH. Coronavirus disease 2019 (COVID-19)! situation report, 88. 2020.

3. Lauer SA, Grantz KH, Bi Q, et al. The incubation period of coronavirus disease 2019 (COVID-19) from publicly reported confirmed cases: estimation and application. Ann Intern Med. 2020;172(9):577-582.

4. Bai Y, Yao L, Wei T, et al. Presumed asymptomatic carrier transmission of COVID-19. JAMA. 2020;323(14):1406-1407.

5. Obstetricians ACo. Gynecologists. COVID-19 FAQs for ObstetricianGynecologists, Gynecology. 